\title{
Diffuse interstellar bands in RAVE survey spectra ${ }^{\star}$
}

\author{
U. Munari ${ }^{1}$, L. Tomasella ${ }^{1}$, M. Fiorucci ${ }^{1}$, O. Bienaymé ${ }^{2}$, J. Binney ${ }^{3}$, J. Bland-Hawthorn ${ }^{4}$, C. Boeche ${ }^{5}$, R. Campbell ${ }^{5,6}$,
} K. C. Freeman ${ }^{7}$, B. Gibson ${ }^{8}$, G. Gilmore ${ }^{9}$, E. K. Grebel ${ }^{10}$, A. Helmi ${ }^{11}$, J. F. Navarro ${ }^{12}$, Q. A. Parker ${ }^{6}$, G. M. Seabroke ${ }^{9,13}$, A. Siebert ${ }^{2,5}$, A. Siviero ${ }^{1}$, M. Steinmetz ${ }^{5}$, F. G. Watson ${ }^{14}$, M. Williams ${ }^{5,7}$, R. F. G. Wyse ${ }^{15}$, and T. Zwitter ${ }^{16}$

1 INAF Osservatorio Astronomico di Padova, Asiago, Italy e-mail: ulisse.munari@oapd.inaf.it

2 Observatoire de Strasbourg, Strasbourg, France

3 Rudolf Pierls Center for Theoretical Physics, University of Oxford, UK

${ }^{4}$ Institute of Astronomy, School of Physics, University of Sydney, Australia

5 Astrophysikalisches Institut Potsdam, Potsdam, Germany

6 Macquarie University, Sidney, Australia

RSAA Mount Stromlo Observatory, Camberra, Australia

8 University of Central Lancashire, Preston, UK

9 Institute of Astronomy, University of Cambridge, UK

10 Astronomisches Rechen-Institut, Center for Astronomy of the University of Heidelberg, Heidelberg, Germany

11 University of Groningen, Groningen, The Netherlands

12 University of Victoria, Victoria, Canada

$13 \mathrm{e} 2 \mathrm{v}$ Centre for Electronic Imaging, School of Engineering and Design, Brunel University, Uxbridge, UK

14 Anglo Australian Observatory, Sydney, Australia

15 Johns Hopkins University, Baltimore, Maryland, USA

16 Faculty of Mathematics and Physics, University of Ljubljana, Ljubljana, Slovenia

Received 21 May 2008 / Accepted 9 July 2008

\section{ABSTRACT}

\begin{abstract}
We have used spectra of hot stars from the RAVE Survey in order to investigate the visibility and properties of five diffuse interstellar bands previously reported in the literature. The RAVE spectroscopic survey for Galactic structure and kinematics records CCD spectra covering the 8400-8800 ̊ wavelength region at 7500 resolving power. The spectra are obtained with the UK Schmidt at the AAO, equipped with the $6 \mathrm{dF}$ multi-fiber positioner. The DIB at $8620.4 \AA$ is by far the strongest and cleanest of all DIBs occurring within the RAVE wavelength range, with no interference by underlying absorption stellar lines in hot stars. It correlates so tightly with reddening that it turns out to be a reliable tool to measure it, following the relation $E_{B-V}=2.72( \pm 0.03) \times E W(\AA)$, valid throughout the general interstellar medium of our Galaxy. The presence of a DIB at $8648 \AA$ is confirmed. Its intensity appears unrelated to reddening, in agreement with scanty and preliminary reports available in the literature, and its measurability is strongly compromised by severe blending with underlying stellar He I doublet at $8649 \AA$. The two weak DIBs at 8531 and $8572 \AA$ do not appear real and should actually be blends of underlying stellar lines. The very weak DIB at $8439 \AA$ A cannot be resolved within the profile of the much stronger underlying hydrogen Paschen 18 stellar line.
\end{abstract}

Key words. ISM: general - ISM: lines and bands - surveys

\section{Introduction}

Diffuse interstellar bands (DIBs) were first discovered by Heger (1922) as stable lines that did not follow the orbital motion seen in spectroscopic binaries. The first systematic studies began only with Merrill (1934, 1936), who noted similarities (occurrence, intensity and velocity) and differences (far wider and with diffuse edges) with respect to atomic interstellar lines. A few years later the first interstellar molecule $(\mathrm{CH})$ was identified by means of absorption lines at $4300.3 \AA$ by Swings \& Rosenfeld (1937). Since then, much progress has been recorded in understanding the absorption spectra of interstellar ions and molecules, while the origin of DIBs is still mysterious after almost a century after their discovery (Sarre 2006). The census of major DIBs in the

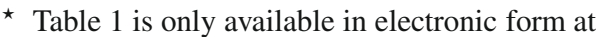
http://www. aanda.org optical region seems quite complete now, at least for those away from strong telluric absorption bands and stellar lines. A compilation maintained by Jenniskens (2007) lists 281 DIBs over the wavelength range from 3980 to $9632 \AA$. DIBs are also observed in external galaxies (e.g. in the SMC by Cox et al. 2007b; in the LMC by Cox et al. 2006; in NGC 1448 by Sollerman et al. 2005; in M 31 by Cordiner et al. 2008), in starbust complexes (Heckman \& Lehnert 2000) and damped Ly- $\alpha$ systems (Jukkarinen et al. 2004; York et al. 2006; Ellison et al. 2008).

The carriers of DIBs are still unknown. Some DIBs tend to show an appreciable correlation with reddening, even if others do not (e.g. Sanner et al. 1978; Krelowski et al. 1999), and this led to the hypothesis that they were produced on or in the interstellar dust grains. However, lack of polarization in DIB profiles (Cox et al. 2007a) argues against, and complex carbon-bearing molecules are generally considered as viable 
carriers (e.g. Fulara \& Krelowski 2000), with fullerenes being subject of intensive laboratory studies (e.g. Herbig 1995; Leach 1995; Iglesias-Groth 2007). PAH (polycyclic aromatic hydrocarbons) have been frequently considered as promising carriers (van der Zwet \& Allamandola 1985; Leger \& Dhendecourt 1985), in particular for their success in explaining the unidentified infrared emission bands (UIR; Sarre et al. 1995). Laboratory studies suggest that only ionized and not neutral PAH could be viable carriers (e.g. Ruiterkamp et al. 2002; Halasinski et al. 2005), and this would agree with the observed insensitivity of DIB intensity to ambient electron density (Gnacinski et al. 2007). A search for correlations between different DIBs has been carried out as a criterion to guide possible identification (a strict correlation may imply a common carrier, whereas a lack of correlation indicates that different species are involved), but the degree of correlation cover the whole interval from good to very poor (e.g. Moutou et al. 1999). Finally, Holmlid (2008) has recently proposed a radically different mechanism for the formation of DIBs, namely doubly excited atoms embedded in the condensed phase named Rydberg matter.

The Radial Velocity Experiment (RAVE) is an ongoing spectroscopic survey of the whole southern sky at galactic latitudes $|b| \geq 25^{\circ}$ for stars in the magnitude interval $9 \leq I_{\mathrm{C}} \leq 12$, with spectra recorded over the $8400-8800 \AA$ range at a resolving power around 7500 . The 150 fiber positioner $6 \mathrm{dF}$ is used at the UK Schmidt telescope of the Anglo-Australian Observatory. The main scientific driver of the project is the study of the stellar kinematics and metallicity of galactic populations away from the galactic plane (e.g. Smith et al. 2007; Seabroke et al. 2008; Veltz et al. 2008). RAVE begun operations in 2003 and has now reached the milestones of the first (Steinmetz et al. 2006) and second (Zwitter et al. 2008) data releases, with a current total of $\sim 250000$ stars already observed.

In this paper we investigate the detectability, measurability and properties of the DIBs known to occur within the RAVE wavelength interval. In this range there is no resonant line from ions sufficiently abundant in the interstellar medium to produce detectable features in high-resolution ground spectra. Weak $\mathrm{C}_{2}$ interstellar lines due to the $(2,0)$ band of the Phillips system are seen longword of hydrogen Paschen 12 and around He I 8777 stellar lines (Gredel \& Muench 1986) in highly reddened stars, the strongest ones occurring at 8751.5, 8753.8, 8761.0, 8763.6, 8773.1 and $8780.0 \AA$ (Gredel et al. 2001).

\section{Target selection}

The flatter is the background stellar continuum, the easier and firmer is the detection and measure of a DIB. Only hot stars provide continua with a sufficiently small number of photospheric lines, and all DIB studies in the literature observed hot stars. Extensive checks with the Castelli \& Munari (2001, hereafter CM01) synthetic spectral atlas, show that spectral types earlier than A7 have - irrespective of the luminosity class - flat background continua at the wavelengths of DIB 8620.4, the principal target of this paper. To be on the safe side by a fairly wide margin, we limited the target selection to A3 and hotter stars.

DIBs generally increase their strength in pace with reddening, which is highest at the lowest galactic latitudes. For this reason, we selected for our analysis only HD stars observed by RAVE at $|b| \leq 10^{\circ}$ as part of calibration runs (normal survey observations are carried out at $|b| \geq 25^{\circ}$ where the reddening is generally negligible and therefore DIB signatures undetectable).

We limited the target selection to HD stars because (i) for all of them an accurate and homogeneous spectral classification has been provided by the Michigan Spectral Survey (Houk \& Cowley 1975; Houk 1978, 1982; Houk \& Smith-Moore 1988; Houk \& Swift 1999); (ii) intrinsic $B-V$ color is known for all spectral types and luminosity classes (Fitzgerald 1970); and (iii) accurate Tycho- $2 B_{\mathrm{T}}$ and $V_{\mathrm{T}}$ photometry is available for all targets. Together, they allow to derive a consistent value for the interstellar reddening affecting each target star.

Two further selection criteria were applied in order to enforce the highest possible quality of the results: we retained only the spectra ( $a$ ) with $S / N \geq 50$ per pixel on the stellar continuum around DIB 8620, and (b) that do not show even the most feeble trace of residual fringing left over by the flat field division. The RAVE Survey CCD is a thinned, back-illuminated one, and as such it naturally presents fringing at the very red wavelengths of RAVE Survey observations. Flat fielding generally provides accurate fringing removal, but in some cases a weak and residual pattern survives (at a level $\leq 1 \%$ that does not affect the main RAVE Survey products: radial velocities and atmospheric parameters of observed stars).

The final target list after application of all above criteria count 68 targets observed by RAVE during the time interval 28 April 2004 to 21 October 2006, eight of them observed twice. They are listed in Table 1 (available electronic only).

\section{DIBs over the RAVE wavelength interval}

A DIB at $8439.4 \AA$ was discovered by Galazutdinov et al. (2000). It is very sharp, and deeply blended with the Paschen 18 photospheric line. It is also intrinsically quite weak, being detectable only in high resolution and high $S / N$ spectra of heavily reddened stars. RAVE spectra cannot reveal it.

A sharp DIB at $8530.7 \AA$ was reported by Jenniskens and Desert (1994), but not confirmed by Galazutdinov et al. (2000). Its wavelength is coincident with the blend of 8528.967, 8529.025, and 8531.508 $\AA$ He I photospheric absorption lines (cf. CM01), compromising any clear detection. The DIB equivalent width reported by Jenniskens \& Desert (1994) for four early type stars characterized by $E_{B-V}$ ranging from 0.30 to 1.28 , shows no clear trend with reddening and instead a better correlation with the intensity of He I expected from the spectral type of the target star. Extrapolating Jenniskens \& Desert (1994) data for HD 183143 reddened by $E_{B-V}=1.28$, an equivalent width of $0.065 \AA$ could be expected for this DIB in the RAVE spectrum of HD 169034 in Fig. 1. This equivalent width is less than half that of the stellar He I blend. If the DIB 8530.7 is real, which we doubt, RAVE spectra cannot disentangle it from the underlying and overwhelming He I $8530 \AA$ absorption blend.

Sanner et al. (1978) listed an uncertain DIB at $8572 \AA$ A. It was dismissed as a photospheric stellar line by Jenniskens \& Desert (1994) and was not detected by Galazutdinov et al. (2000). CM01 atlas shows the presence of a weak and diffuse blend of many photospheric absorption lines centered at the same wavelength as the supposed DIBs, confirming the proposed DIB as a spurious detection.

A moderately strong, broad and complex profile DIB, loosely centered at $8648.3 \AA$, was discovered by Sanner et al. (1978). It was later confirmed by Herbig \& Leka (1991), Jenniskens \& Desert (1994) and Wallerstein et al. (2007). Over the wavelength interval covered by the 8650 DIB there are two strong He I photospheric absorption lines at 8648.258 and 8650.811 that appreciably confuse the picture (cf. CM01). RAVE spectra support the presence of a DIB at these wavelengths, whose intensity however does not correlate at all with 


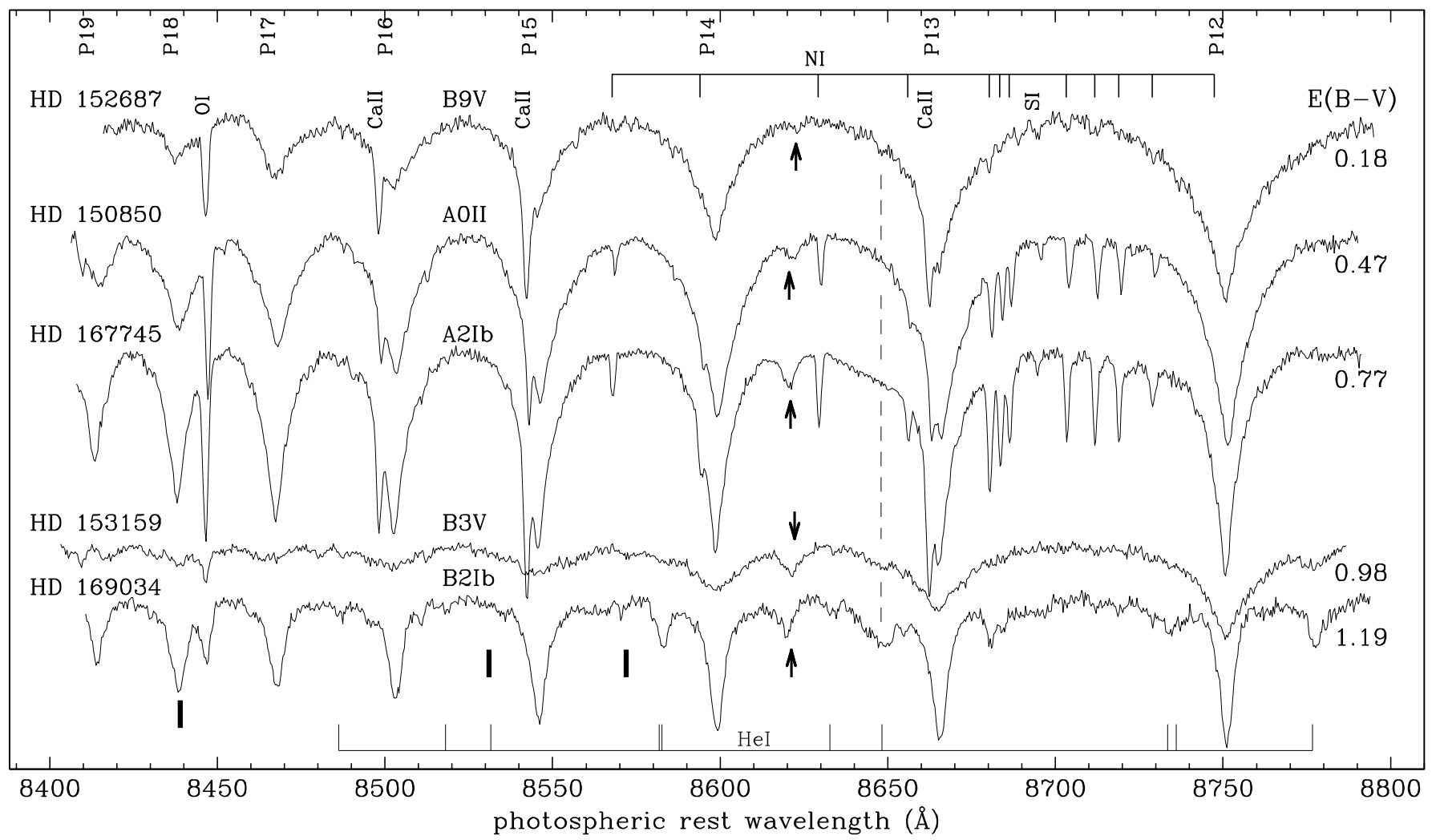

Fig. 1. A sample of RAVE spectra of early type HD stars ordered according to reddening. The strongest stellar lines are identified. The arrows point to DIB 8620, the thick vertical marks to DIBs 8439, 8531, 8572, and the dashed line to DIB $8648 \AA$.

reddening (as already noted by Sanner et al. 1978, their Fig. 4). In fact, in the RAVE spectra of Fig. 1, the 8648.3 DIB is clearly present at $E_{B-V}=0.18$, missing at $E_{B-V}=0.47$, prominent and broader at $E_{B-V}=0.77$, feeble or absent at $E_{B-V}=0.98$, and again strong at $E_{B-V}=1.19$. In the most reddened spectrum of Fig. 1 (HD 169034), the expected intensity of the interfering $\mathrm{He}$ I blend at $8648.258,8650.811$ is equal to the intensity of the nearby He I blend at $8581.856,8582.670$, which lead us to speculate that the equivalent width of the 8648.3 DIB in this spectrum should be $\sim 0.25 \AA$. A very close match is provided by Wallerstein et al.'s (2007) spectra for the stars HD 169454 and HD 183143 (their Fig. 1). Given the obvious interference by strong underlying stellar He I and the lack of an appreciable correlation with reddening, we will not further discuss the 8648.3 DIB as seen in RAVE spectra.

\section{The 8620 A DIB}

The strongest DIB over the RAVE wavelength range appears at $8620.4 \AA$. It was first discovered by Geary (1975), and then confirmed by all later investigators. Its tight correlation with reddening was discovered and discussed by Munari (2000, hereafter M00), and later confirmed by Wallerstein et al. (2007).

\subsection{DIB measurement}

The equivalent width and heliocentric wavelength of the 8620 DIB have been obtained on RAVE spectra by integrating - over the wavelength range of the DIB - the difference between the extrapolated underlying continuum and the observed spectrum affected by the DIB. The underlying continuum has been fitting with a 6 th order Lagrange polynomial between the
Paschen 13 and 14 line centers. The results are reported in Table 1.

Eight of the sixty-eight program stars have a second RAVE spectrum satisfying the quality selection criteria outlined in Sect. 2. Four of them have been obtained with similar instrument set-ups (plate and fiber) on adjacent nights, while the other four were observed with different set-ups one year apart. The mean difference between the two measurements of the equivalent width in these eight pairs is:

$\sigma(E W)=0.014 \AA$

which we consider to be representative of the mean accuracy of our DIB measurements.

The intrinsic DIB wavelength is reported as $8620.8 \AA$ by Galazutdinov et al. (2000) from observations toward a single star (HD 23180), and as 8621.2 ^ by Jenniskens \& Desert (1994) from observations toward four stars. M00 gives $8620.4 \AA$ from observations of 37 northern stars in the general Galactic anticenter direction, after correction for the velocity of interstellar atomic lines (Na I and K I). The RAVE program stars lies toward the Bulge and close to the Galactic center (see galactic coordinates in Table 1 and Fig. 3). Adopting the Brand \& Blitz (1993) maps for the radial velocity of interstellar medium, the average velocity of the medium along the lines of sight to the program stars is essentially null. Therefore, the mean of heliocentric DIB wavelength in Table 1 represents also the intrinsic barycentric wavelength, whose average value is:

$\lambda(\mathrm{DIB})=8620.4( \pm 0.1) \AA$. 


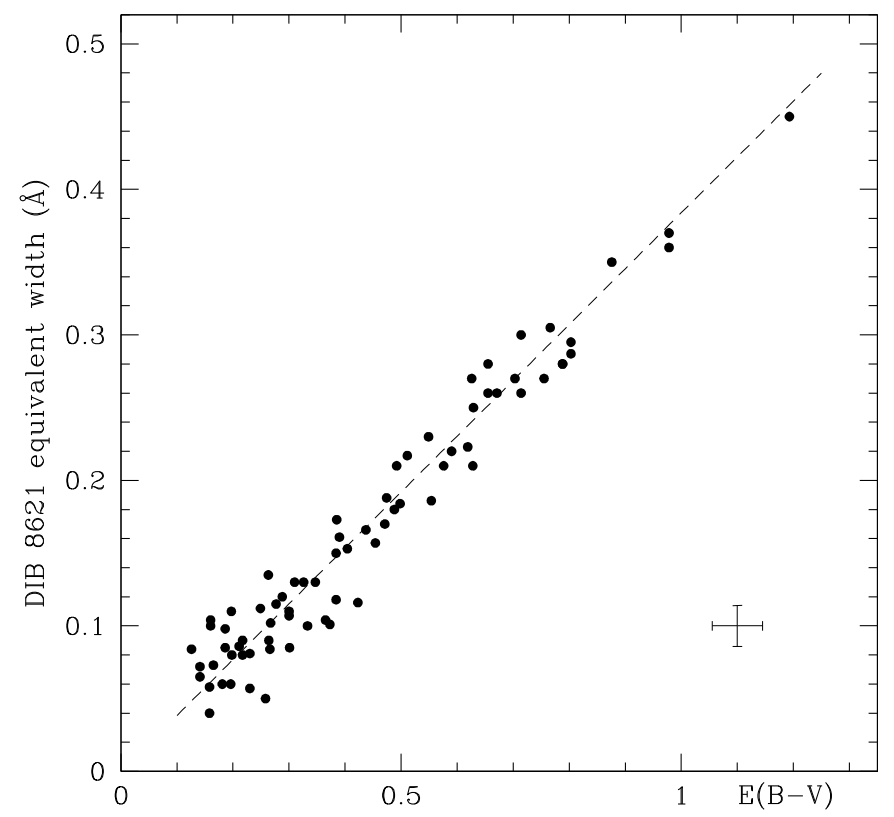

Fig. 2. The equivalent width of the diffuse interstellar band at $8620.4 \AA$ as function of reddening. The dashed line represents Eq. (4) and the error bars are the average uncertainties of plotted points from Eqs. (1) and (3).

\subsection{Reddening of program stars}

The reddening of the sixty-eight program stars was homogeneously derived from the Michigan spectral type of the HD stars, the corresponding intrinsic color $(B-V)_{\mathrm{J}}$ from Fitzgerald (1970), and observed the Tycho- $2(B-V)_{\mathrm{T}}$ color ported to the Johnson system via Bessell (2000) transformations (cf. Sect. 2). The reddening derived for the program stars is listed in Table 1 together with their spectro-photometric parallax derived adopting absolute $M_{V}$ magnitudes from the Michigan Project ${ }^{1}$. The individual error sources contributing to the overall reddening error are: (i) the natural color width of a spectral sub-type (on the average 0.020 mag for O-A3 stars); (ii) the uncertainty in the spectral classification (if taken equivalent to one spectral subclass, it is also $0.020 \mathrm{mag}$ ); (iii) the error of Tycho-2 $(B-V)_{\mathrm{T}}$ color (on average $0.034 \mathrm{mag}$ ); (iv) the uncertainty of the color transformation from Tycho-2 to the Johnson system (unknown, and assumed to amount to a mere $0.010 \mathrm{mag}$ ). Considering them as independent quantities and adding them in quadrature, the mean value of the overall error budget of reddening determination is:

$\sigma\left(E_{B-V}\right)=0.045 \mathrm{mag}$.

\subsection{A tight relation between reddening and equivalent width}

Figure 2 illustrates the relation between reddening and equivalent width of DIB 8620.4 for the seventy-six spectra of the sixty-eight program stars of Table 1 . The relation is remarkably straight, with a least square fitting of:

$E_{B-V}=2.72( \pm 0.03) \times E W$

where the $E W$ is expressed in $\AA$. The rms of the points is $0.020 \AA$ in $E W$ and 0.053 mag in $E_{B-V}$, which are only modestly larger than the typical measurement error in both axes as given by

\footnotetext{
${ }^{1}$ http://www.astro.lsa.umich.edu/users/hdproj/ mosaicinfo/ absmag.html
}

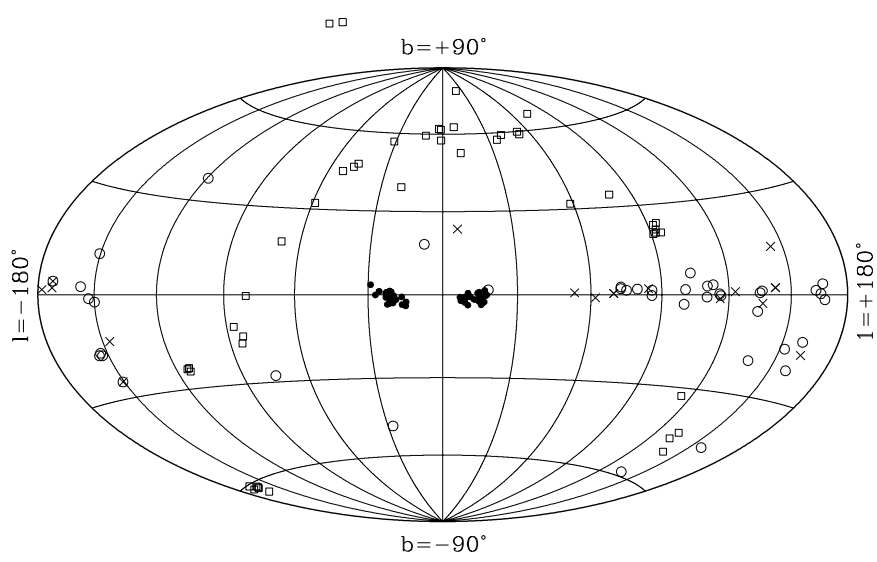

Fig. 3. Aitoff projection in galactic coordinates of the program stars (filled circles). Also plotted are the stars studied by Munari (2000, open circles), and those observed by Jenniskens \& Desert (1994), Sanner et al. (1978, crosses) and Wallerstein et al. (2007, squares).

Eqs. (1) and (3). This argues in favor of a very small intrinsic scatter of the points around Eq. (4), a remarkable property already preliminary focused upon by M00. The absence of significant cosmic scatter in the proportionality between reddening and equivalent width of the DID 8620 has two main implication: (a) the DIB carrier has an intimate partnership with the solid phase of the interstellar medium. A search and study of the polarization across the DIB profile would be worthwhile, and (b) the DIB 8620.4 can now be considered a viable tool to actually measure the amount of reddening and not simply to guess its presence.

The dispersion of the points along the mean relation in Fig. 2 is slightly larger at lower reddenings: the rms in $E W$ of the points with $E W \leq 0.14 \AA$ is $0.022 \AA$, and $0.016 \AA$ for $E W \geq 0.14 \AA$. While the significance of this small difference is uncertain given the small number statistics, for sake of discussion it could be argued that some cosmic scatter - even if marginal - is actually present in the relation between reddening and intensity of the DIB 8620.4. In fact, a sharper relation at increasing $E_{B-V}$ could simply mean than one starts to sample multiple clouds in the line-of-sight and thus any deviations between single clouds will be somewhat averaged out. Wallerstein et al. (2007) reported that stars seen through the $\rho$ Oph molecular cloud show a DIB weaker than expected.

The proportionality relation found by M00 from 37 northern stars observed at high resolution and high $S / N$ is $E_{B-V}=2.69( \pm 0.03) \times E W$, which is essentially identical to Eq. (4). This is remarkable because the CCD adopted by M00 for his Echelle observations was a thick, front illuminated one without any fringing, and therefore ideal to aim for the highest accuracy. Fitting the data of the four stars observed by Jenniskens $\&$ Desert (1994) in high resolution with a Reticon detector gives $E_{B-V}=2.77( \pm 0.1) \times E W$, and the 10 stars observed by Sanner et al. (1978) at low resolution again with a Reticon detector provides $E_{B-V}=2.76( \pm 0.06) \times E W$. Finally, when the reddening of the target stars is homogeneously computed in the same way as done for this paper, also the Wallerstein et al. (2007) data support Eq. (4) above (Wallerstein, private communication).

Our study and data from these other investigations cover about two hundreds different stars distributed over a great range of distances from the Sun and over a wide range Galactic longitudes, from the Galactic anti-center mapped by M00 to the Galactic center in this paper, as illustrated by Fig. 3. These stars have been observed with quite different techniques and 
instruments, and the equivalent width of the DIB measured with different approaches. Yet, they define one and the same proportional relation. We therefore propose that Eq. (4) can be safely adopted as a direct way to derive the reddening caused by the general interstellar medium.

In general terms, it could be argued that proportionality relation between reddening and DIB intensity could take the form $E_{B-V}=\alpha(l, b, d) \times E W$, where $\alpha$ is allowed to vary as function of galactic coordinates and distance, reflecting local inhomogeneities in the interstellar medium. The calibration of $\alpha$ requires observations of stars scattered through the whole Galaxy in a number which is orders of magnitudes larger than currently available. As noted by M00, only the forthcoming ESA's GAIA mission will be in a position to provide such a large dataset, both in the form of accurate distances and spectra covering the DIB 8620.4 in high resolution.

Acknowledgements. We would like to thank George Wallerstein and Karin Sandstrom for useful discussions, and the anonymous referee for effective comments. The spectra here used were obtained as part of the RAVE survey using the UK Schmidt Telescope operated by the Anglo-Australian Observatory. The RAVE project is managed and supported by the Astrophysikalisches Institut Potsdam, Anglo-Australian Observatory, Australian National University, University of Basel, University of Cambridge, University of Edinburgh, University of Heidelberg, Johns Hopkins University, University of Ljubljana, Macquarie University, University of Oxford, INAF Astronomical Observatory of Padova, Steward Observatory, Swinburne University, University of Utrecht, University of Victoria. The RAVE web site is at www .rave-survey.org

\section{References}

Bessell, M. S. 2000, PASP, 112, 961

Brand, J., \& Blitz, L. 1993, A\&A, 275, 67

Castelli, F., \& Munari, U. 2001, A\&A, 366, 1003 (CM01)

Cordiner, M. A., Cox, N. L. J., Trundle, C., et al. 2008, A\&A, 480, L13

Cox, N. L. J., Cordiner, M. A., Cami, J., et al. 2006, A\&A, 447, 991

Cox, N. L. J., Boudin, N., Foing, B. H., et al. 2007a, A\&A, 465, 899

Cox, N. L. J., Cordiner, M. A., Ehrenfreund, P., et al. 2007b, A\&A, 470, 941

Ellison, S. L., York, B. A., Murphy, M. T., et al. 2008, MNRAS, 383, L30

Fitzgerald, M. P. 1970, A\&A, 4, 234

Fulara, J., \& Krelowski, J. 2000, New Astr. Rev., 44, 581

Galazutdinov, G. A., Musaev, F. A., Krelowski, J., \& Walker, G. A. H. 2000, PASP, 112, 648
Geary, J. C. 1975, Ph.D. Thesis, Univ. Arizona

Gnacinski, P., Sikorski, J. K., Galazutdinov, G. A., et al. 2007, A\&A, 469, 201

Gredel, R., \& Muench, G. 1986, A\&A, 154, 336

Gredel, R., Black, J. H., \& Yan, M. 2001, A\&A, 375, 553

Halasinski, T. M., Salama, F., Allamandola, L. J., et al. 2005, ApJ, 628, 555

Heckman, T. M., \& Lehnert, M. D. 2000, ApJ, 537, 690

Heger, M. L. 1922, LickOB, 10, 141

Herbig, G. H. 1995, ARA\&A, 33, 19

Herbig, G. H., \& Leka, K. D. 1991, ApJ, 382, 193

Holmlid, L. 2008, MNRAS, 384, 764

Houk, N. 1978, Michigan Catalogue of Two-Dimensional Spectral Types for the HD Stars, Vol. 2 (Ann Arbor: Univ. Michigan)

Houk, N. 1982, Michigan Catalogue of Two-Dimensional Spectral Types for the HD Stars, Vol. 3 (Ann Arbor: Univ. Michigan)

Houk, N., \& Cowley, A. P. 1975, Michigan Catalogue of Two-Dimensional Spectral Types for the HD Stars, Vol. 1 (Ann Arbor: Univ. Michigan)

Houk, N., \& Smith-Moore, M. 1988, Michigan Catalogue of Two-Dimensional Spectral Types for the HD Stars, Vol. 4 (Ann Arbor: Univ. Michigan)

Houk, N., \& Swift, C. 1999, Michigan Catalogue of Two-Dimensional Spectral Types for the HD Stars, Vol. 5 (Ann Arbor: Univ. Michigan)

Iglesias-Groth, S. 2007, ApJ, 661, L167

Jenniskens, P. 2007, http://leonid.arc.nasa.gov/DIBcatalog.html

Jenniskens, P., \& Desert, F. X. 1994, A\&AS, 106, 39

Junkkarinen, V. T., Cohen, R. D., Beaver, E. A., et al. 2004, ApJ, 614, 658

Krelowski, J., Ehrenfreund, P., Foing, B. H., et al. 1999, A\&A, 347, 235

Leach, S. 1995, P\&SS, 43, 1153

Leger, A., \& Dhendecourt, L. 1985, A\&A, 146, 81

Merrill, P. W. 1934, PASP, 46, 206

Merrill, P. W. 1936, ApJ, 83, 126

Moutou, C., Krelowski, J., D’Hendecourt, L., \& Jamroszczak, J. 1999, A\&A, 351,680

Munari, U. 2000, in Molecules in Space and in the Laboratory, ed. I. Porceddu, \& S. Aiello, It. Phys. Soc. Conf. Proc., 67, 179 (M00)

Ruiterkamp, R., Halasinski, T., Salama, F., et al. 2002, A\&A, 390, 1153

Sanner, F., Snell, R., \& Vanden Bout, P. 1978, ApJ, 226, 460

Sarre, P. J. 2006, J. Moc. Spc, 238, 1

Sarre, P. J., Miles, J. R., \& Scarrott, S. M. 1995, Science, 269, 674

Seabroke, G. M., Gilmore, G., Siebert, A., et al. 2008, MNRAS, 384, 11

Smith, M. C., Ruchti, G. R., Helmi, A., et al. 2007, MNRAS, 379, 755

Sollerman, J., Cox, N., Mattila, S., et al. 2005, A\&A, 429, 559

Steinmetz, M., Zwitter, T., Siebert, A., et al. 2006, AJ, 132, 1645

Swings, P., \& Rosenfeld, L. 1937, ApJ, 86, 483

van der Zwet, G. P., \& Allamandola, L. J. 1985. A\&A, 146, 76

Veltz, L., Bienaym, O., Freeman, K. C., et al. 2008, A\&A, 480, 753

Wallerstein, G., Sandstrom, K., \& Gredel, R. 2007, PASP, 119, 1268

York, B. A., Ellison, S. L., Lawton, B., et al. 2006, ApJ, 647, L29

Zwitter, T., Siebert, A., Munari, U., et al. 2008, AJ, 136, 421 


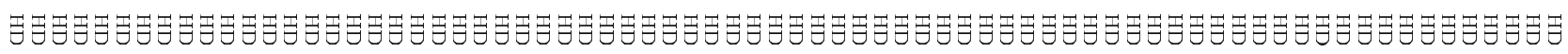

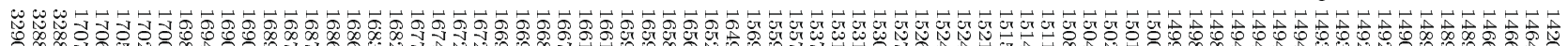

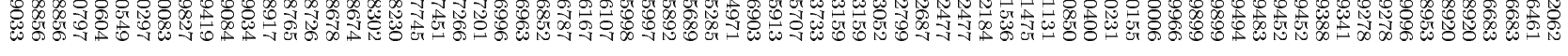

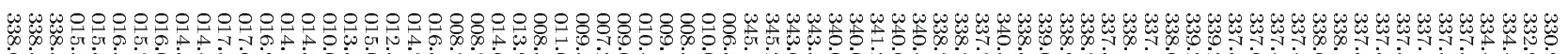

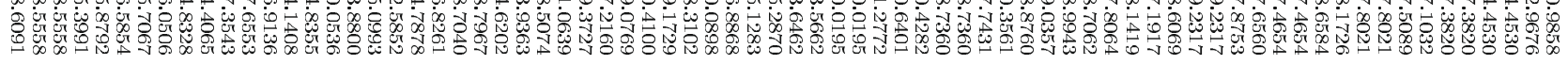

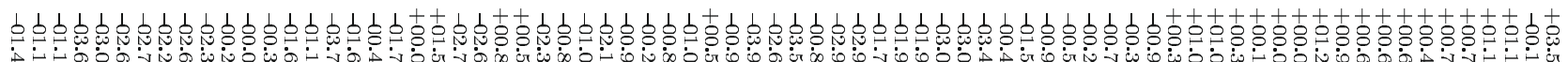

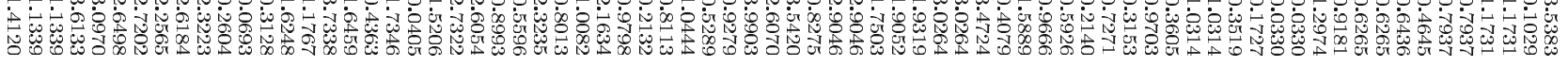

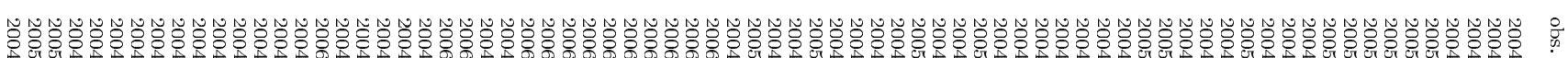

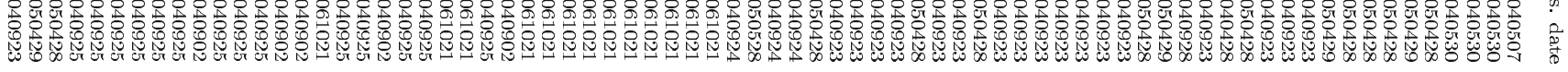

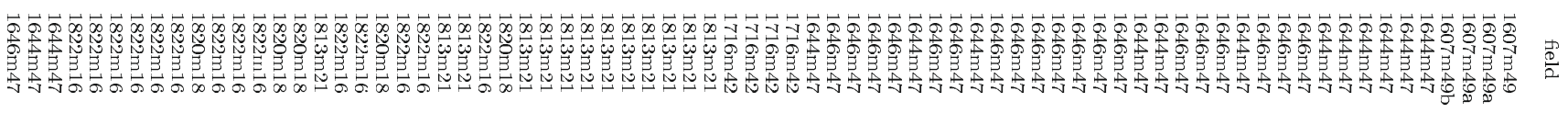

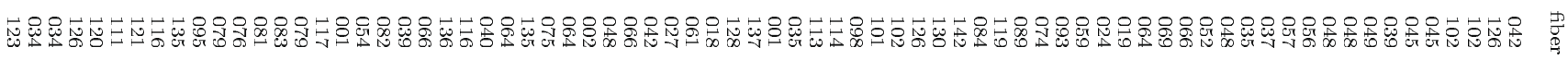

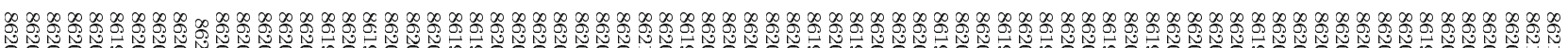

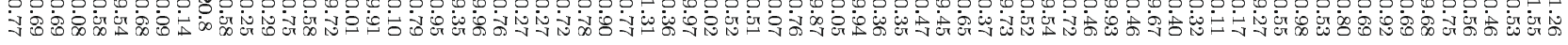
$00000000000000000000000000000000000000000000000000000000000000000000000000 \%$

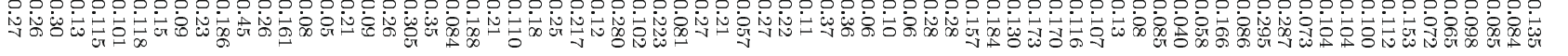

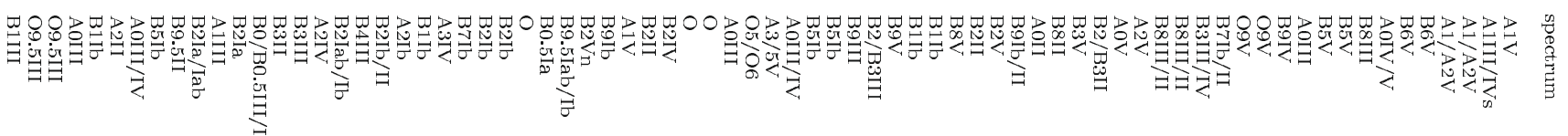

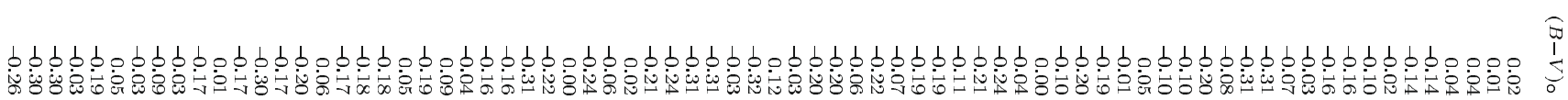

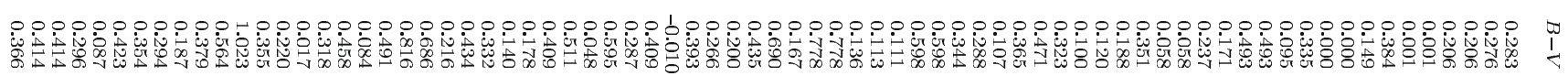

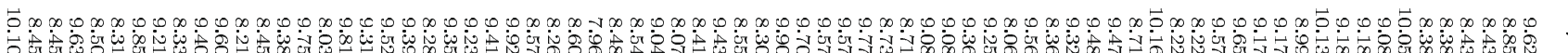

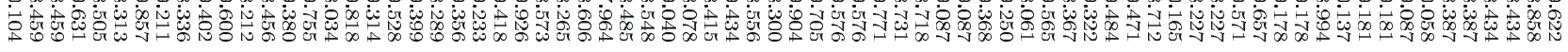

ئ

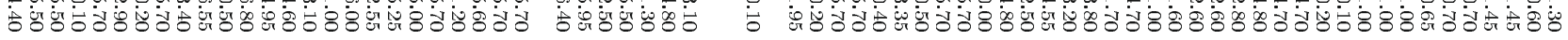

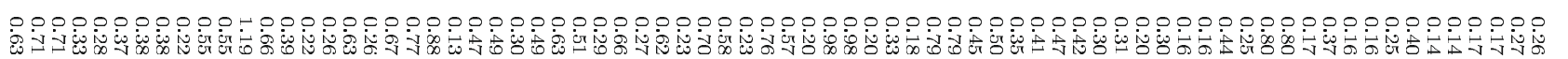

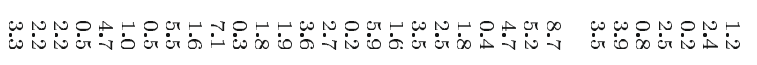

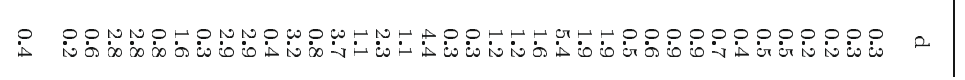

